\title{
Nutritional Deficiencies in Patients after Roux-en-Y Gastric Bypass and Sleeve Gastrectomy during 12-Month Follow-Up
}

\author{
Aleksander Antoniewicz ${ }^{1}$ (D) P Piotr Kalinowski ${ }^{1} \cdot$ Kamila J. Kotulecka $^{1} \cdot$ Piotr Kocoń $^{1} \cdot$ Rafał Paluszkiewicz $^{1}$. \\ Piotr Remiszewski ${ }^{1} \cdot$ Krzysztof Zieniewicz $^{1}$
}

Published online: 14 June 2019

(C) The Author(s) 2019

\begin{abstract}
Introduction Roux-en-Y gastric bypass (RYGB) and laparoscopic sleeve gastrectomy (LSG) are the two most frequently performed bariatric operations. These two types of metabolic surgery alter the anatomy and function of digestive tract producing significant weight loss in morbidly obese patients but may lead to malnutrition.

Aim Analysis of incidence and severity of malnutrition after bariatric surgery in patients submitted to RYGB or LSG during 12 months of follow-up.

Material and Methods Retrospective study of 98 patients after RYGB $(n=47)$ or LSG $(n=51)$ assessed for nutritional deficiencies during 12 months after surgery was conducted. The differences in body mass index (BMI) and blood tests including erythrocytes, haemoglobin, total protein, albumin, iron, ferritin, transferrin, vitamin B12, folic acid, calcium and phosphorus concentrations were compared between groups before the operations and at 1 and 12 months.

Results Nutritional deficiencies were common before surgery with prevalence up to $19.6 \%$ for albumin in the LSG group. Median preoperative BMI levels and albumin concentrations were higher in the RYGB group compared to the LSG group, but there was no difference in percent excess weight loss (\%EWL) at 1 and 12 months between LSG and RYGB. One month after LSG erythrocyte count, haemoglobin, iron, ferritin and transferrin levels were significantly higher than in the RYGB group. These differences subsided at 12 months. At 12 months, only the prevalence of vitamin $\mathrm{B}_{12}$ deficiency was significantly higher in the RYGB group.
\end{abstract}

Conclusion Both RYGB and LSG lead to nutritional deficiencies despite different properties of operations and similar \%EWL during follow-up.

Keywords Obesity $\cdot$ Malnutrition $\cdot$ Nutritional deficiencies $\cdot$ Bariatric surgery $\cdot$ Sleeve gastrectomy $\cdot$ Gastric bypass

\section{Introduction}

Obesity in Europe affects 10-30\% adults (http://www.euro. who.int/en/health-topics/noncommunicable-diseases/obesity/ data-and-statistics); therefore, it can be regarded as a major healthcare problem of the twenty-first century. It has been proven that surgery is the best treatment for morbid obesity [1]. The most commonly performed bariatric procedures are Roux-en-Y gastric bypass (RYGB) and laparoscopic sleeve gastrectomy (LSG) [2]. Bariatric operations used to be
Aleksander Antoniewicz

aleksander.antoniewicz@gmail.com

Piotr Kalinowski

kalip@o2.pl

Kamila J. Kotulecka

kamila.kotulecka@gmail.com

Piotr Kocoń

piotrkocon93@gmail.com
Rafał Paluszkiewicz

jrpalusz@gmail.com

Piotr Remiszewski

remi@wp.pl

Krzysztof Zieniewicz

krzysztof.zieniewicz@wum.edu.pl

1 Department of General, Transplant and Liver Surgery, Medical University of Warsaw, Warsaw, Poland 
classified as purely restrictive, restrictive/malabsorptive and primarily malabsorptive [3]. Roux-en-Y gastric bypass was considered a method combining restriction and malabsorption and sleeve gastrectomy at the time of its development was added as a restrictive component to a biliopancreatic diversion [4]. As more scientific data was gathered, these two common types of procedures were classified as restrictive [5]. Then even more important studies were published showing the role of gastrointestinal hormones in mechanisms of action of bariatric procedures and the focus was changed to metabolic surgery. However, both RYGB and SG cause significant weight reduction in morbidly obese patients, but may also lead to malnutrition [6].

Considerable amount of data showing risk of nutritional deficiencies after RYGB is available and recently, studies regarding LSG have also been published. There are still disputes not only regarding type of surgery, but also the individual variations in techniques employed.

LSG is considered a safe procedure due to a low risk of nutritional deficiencies and gastric bypass is associated with increased nutritional deficiencies risk profile due to its complexity and alteration of gastrointestinal anatomy. In a recent analysis of a large health care database focusing on anaemia related to micronutrient deficiency, it was found more frequently in RYGB patients [7].

Most recent studies comparing LSG and RYGB prove that these types of operation may lead to similar weight reduction effect but cause different micronutritional deficiencies [8-11]. The assessment of general nutritional status of morbidly obese patients cannot be done using common nutritional screening tools for non-morbidly obese individuals due to different characteristics of malnutrition in those groups.

The aim of our study was to analyse the incidence and severity of malnutrition in bariatric patients before surgery and during 12 months of follow-up after different types of bariatric operations (RYGB and LSG).

\section{Patients and Methods}

The data of 322 patients who underwent bariatric operations at our centre between 2001 and 2014 was reviewed. Retrospective analysis of 98 bariatric patients was done with focus on early results at 1-month and 1-year follow-up data. There were 51 LSG and 47 RYGB patients who underwent operation and had complete 1-year nutritional follow-up. The indications for surgery were body mass index (BMI) $\geq 40 \mathrm{~kg} /$ $\mathrm{m}^{2}$ or BMI $\geq 35 \mathrm{~kg} / \mathrm{m}^{2}$ with comorbidities, age $18-60$ years. The same indications were applicable to both types of procedures and there were no additional selection criteria.

The exclusion criteria were poorly controlled medical or psychiatric disorders, active alcohol or substance abuse, active duodenal/gastric ulcer disease, large hiatal hernia, previous major gastrointestinal surgery and malignancy. Body mass index was calculated as weight in kilogrammes divided by height in meters squared. The percentage of excess weight loss (\%EWL) was defined as weight lost divided by preoperative excess weight above the upper limit of the ideal weight at BMI $25 \mathrm{~kg} / \mathrm{m}^{2}$.

\section{Surgical Technique}

In RYGB, a small gastric pouch (10-20 ml) was formed by division of the stomach with linear stapler (TA90B, medium/ thick reload, Medtronic). It was anastomosed side to side on the anterior wall with an alimentary loop of $100 \mathrm{~cm}$ in length that was created $100 \mathrm{~cm}$ distally from the ligament of Treitz.

In LSG, calibration of the stomach was done on the 36F gastric tube and resection was completed using linear stapler (60-mm and 45-mm long, EndoGIA, medium/thick reload, Covidien).

\section{Follow-up}

The follow-up visits took place at 1 and 12 months after surgery. Weight loss was assessed and blood tests were done before surgery and at every follow-up appointment. Data chosen for the evaluation of nutritional status included the following: erythrocyte count, haemoglobin, serum protein, albumin, iron, ferritin, transferrin, vitamin $B_{12}$, folic acid and calcium concentrations. At discharge, all patients received daily multivitamin supplementation (Centrum®), Pfizer Trading Polska; vitamin A $800 \mu \mathrm{g}$, lutein $500 \mu \mathrm{g}$, vitamin E $15 \mathrm{mg}$, vitamin C $100 \mathrm{mg}$, vitamin K $30 \mu \mathrm{g}$, vitamin $B_{1} 1.4 \mathrm{mg}$, vitamin $B_{2} 1.75 \mathrm{mg}$, vitamin $B_{6} 2 \mathrm{mg}$, vita$\min \mathrm{B}_{12} 2.5 \mu \mathrm{g}$, vitamin D $5 \mu \mathrm{g}$, biotin $62.5 \mu \mathrm{g}$, folic acid $200 \mu \mathrm{g}$, niacin $20 \mathrm{mg}$, pantothenic acid $7.5 \mathrm{mg}$, calcium $162 \mathrm{mg}$, phosphorus $125 \mathrm{mg}$, magnesium $100 \mathrm{mg}$, zinc $5 \mathrm{mg}$, iodine $100 \mu \mathrm{g}$, manganese $2 \mathrm{mg}$, chromium $4 \mu \mathrm{g}$, molybdenum $50 \mu \mathrm{g}$, selenium $30 \mu \mathrm{g}$, iron $5 \mathrm{mg}$ ), daily $100 \mathrm{mg}$ of iron(III)hydroxide polymaltose complex and $1 \mathrm{mg}$ of sublingual vitamin $\mathrm{B}_{12}$ every 4 weeks.

\section{Statistical Analysis}

Data are presented as median with interquartile range. The variables were compared prior to and after the operation with Kruskal-Wallis, Mann-Whitney $U$ test, ANOVA and Friedman tests where applicable. The excesses and deficiencies of parameters with reference to the laboratory norms of the hospital were calculated with chi-squared test. Correlations between variables were assessed using 2-tailed Pearson's correlation test. All reported $p$ values are significant at $<0.05$. Statistical analysis was performed using Statistica 13.1 (Statsoft). 


\section{Results}

Both study groups were comparable at baseline in age and gender (Table 1). Women were the majority of patients in both operation groups. The mean pre-operative BMI and albumin level were significantly higher in the RYGB group compared to the LSG group. Pre-operatively, $19.6 \%$ and $2.1 \%$ of patients had hypoalbuminemia in LSG and RYGB groups, respectively.

A significant BMI reduction was observed in both groups at 1 and 12 months of the follow-up. The mean BMI values were significantly lower in the LSG group compared to RYGB during the whole follow-up, but the differences in $\%$ EWL were not significant (Table 2). At 1 month postoperatively, there was a significant difference between groups in erythrocyte count, haemoglobin, total protein, iron, ferritin and calcium levels. One-year follow-up results were significantly different for vitamin $\mathrm{B}_{12}$, calcium and BMI. Although for some parameters, such as haemoglobin, iron, transferrin and ferritin, there was no significant difference between baseline and twelve postoperative month values and there were transient alterations during early postoperative period.

It is worth mentioning that $20.3 \%$ of patients had abnormally low ferritin levels preoperatively and the prevalence of this deficit increased to $30.6 \%$ postoperatively. It was not related to the type of operation (Table 3). Preoperatively, 37\% of patients had anaemia which prevalence increased to $43.4 \%$ postoperatively. Median serum concentrations of vitamin $\mathrm{B}_{12}$ were significantly lower in the RYGB group than in the LSG group 12 months postoperatively. The calcium concertation increased during follow-up in the LSG group and in 1-month and 12-month follow-up, the difference between both groups became statistically significant. The incidence of vitamin $\mathrm{B}_{12}$ deficiency has quadrupled (6.4\% to $25.5 \%$ ) during the follow-up period after RYGB. One year after LSG, $13.7 \%$ patients developed abnormally low level of folic acid with no patients presenting that deficiency preoperatively. In the early postoperative period, transferrin and total protein deficiencies were observed in the whole study group and were only partially corrected at 12 months (Table 3). No significant correlations were found between analysed variables and \%EWL after surgery. The analysis of multiple deficiencies present both before and 12 months after

Table 1 Patients' characteristics at baseline

\begin{tabular}{lllr}
\hline & RYGB $(n=47)$ & LSG $(n=51)$ & $p$ \\
\hline Female sex \% & $70.21 \%$ & $65.96 \%$ & 0.65 \\
Age & $44.29(8.97)$ & $43.93(11.00)$ & 0.87 \\
BMI & $49.78(46.25-55.63)$ & $44.11(41.27-49.12)$ & $<0.05$
\end{tabular}

$R Y G B$, Roux-Y gastric bypass; $L S G$, laparoscopic sleeve gastrectomy; $B M I$, body mass index bariatric operations was also performed. It showed that in the LSG group, 6 (11.8\%), 3 (5.9\%), 1 (2.0\%), 0 (0.0\%) and 1 (2.0\%) patients had $1,2,3,4$ or 5 (out of 12 parameters) persisting deficiencies respectively. In the RYGB group, the numbers were $8(17.0 \%), 3(6.4 \%), 0(0.0 \%), 2(4.3 \%)$ and 0 $(0.0 \%)$ patients, respectively.

\section{Discussion}

Prior to surgery, micronutritional and protein deficiencies were common in both patients' groups. The policy adopted in our centre was to start supplementation immediately after surgery and continue it indefinitely. As there is a lack of strict pre- and perioperative correction recommendations, the authors believed that it was not required to correct the existing deficiencies before intervention and that the postoperative treatment is enough to correct and maintain adequate micronutrient levels. It is an acceptable and common approach as reported by Peterson et al. in the article describing nutritional status of bariatric patients prior to surgery [12]. Alexandrou et al. in a review describing nutritional deficiencies in obesity and after bariatric surgery reported that this kind of surgery may exacerbate them. Therefore, it was recommended to encourage patients to supplement deficient nutrients at baseline and after surgery [9]. Muñoz et al. suggested that biochemical preoperative assessment in both groups of patients is necessary due to an increased risk of development of various deficiencies [13]. Jáuregui-Lobera in a review of iron status after bariatric surgery advised to correct iron deficiencies preoperatively and during follow-up in order to prevent anaemia [14]. It is worth mentioning that patients who partially lose excess weight prior to the surgery are more motivated to obey all the regimes after operation, including changes of lifestyle and habits as was reported by Gerber et al. [15]. According to the van der Beek et al. study describing nutritional status of patients undergoing gastric bypass, the malnutrition status should be assessed and deficiencies treated prior to the surgery. It may not only prolong the post-operative period of optimal nutritional status, but also prevent patients from developing any new micronutrient deficiencies [16].

Both types of bariatric operations lead to significant weight loss, but the superiority of one type over another is disputable. Manning et al. reported better weight loss after RYGB [17]. However, in our study, the difference between \%EWL at each follow-up was not significant. A recent meta-analysis of nine clinical trials did not show a significant difference in weight loss and \%EWL between these operations [18].

Bariatric surgery may lead to alterations in calcium levels due to an increased bone remodelling after operation [19]. In a review describing bone and mineral metabolism in patients undergoing Roux-en-Y gastric bypass, Hage et al. suggested that fluctuations of calcium levels can be a result of decreased 


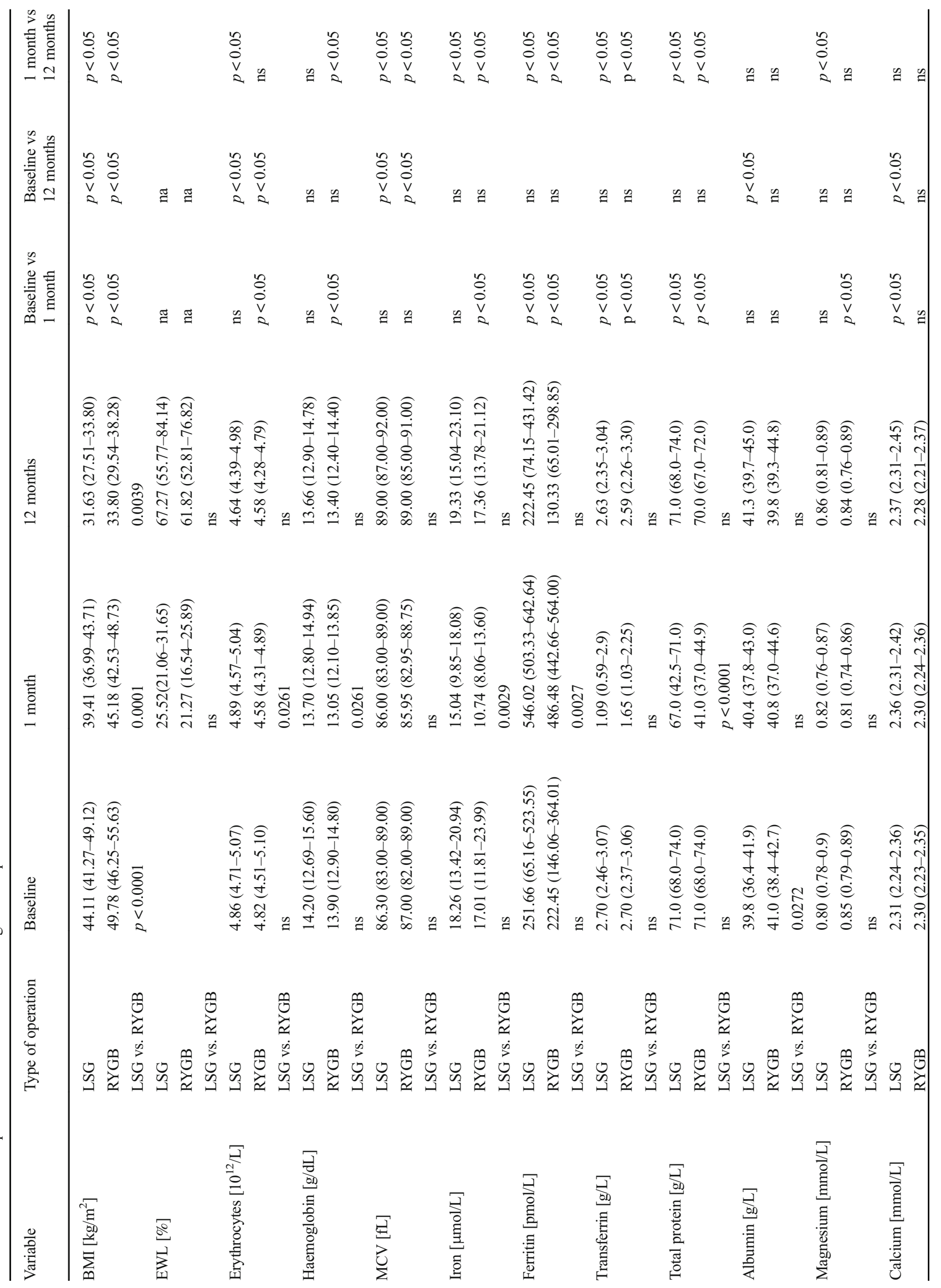


mechanical bones loading that occurs postoperatively as a result of weight loss. Another possible mechanisms are malabsorption and secondary changes in calciotropic hormones or gut and adipose tissue hormone alterations [20]. In our study, calcium deficiencies were also present, but the number of these cases was limited.

The two major factors responsible for anaemia presenting in $15-20 \%$ of our patients were deficiencies in iron and vita$\min B_{12}$. The major fluctuations of erythrocyte count, mean corpuscular volume and haemoglobin, iron, ferritin and transferrin concentrations would imply that both RYGB and LSG cause alterations in erythropoiesis and iron metabolism. Although many of the reported differences are statistically significant, the clinical relevance sometimes remains unclear. It is vital to notice, that at 1 month of follow-up high transferrin deficiency rates ( $63.2 \%$ in our cohort) accompanied by $0 \%$ ferritin deficiency do not result in an increase in iron serum concentrations or haemoglobin levels in both groups. These features often occur in anaemia caused by inflammation/ chronic disease. In our study, the percentage of patients with anaemia (haemoglobin deficiency) remained fairly stable in 12-month follow-up. Another explanation for lower haemoglobin levels is menstrual bleeding among women in premenopausal age, which may lead to disproportion between lost and absorbed iron [9, 21-24]. The majority of patients in our study were menstruating women which can contribute to an increased anaemia rates in this group of bariatric patients. However, male patients are also at risk of anaemia. In a crosssectional study by Lefebvre et al., preoperative haemoglobin deficiencies were present in $6.0 \%$ of women and $6.6 \%$ of men, for our cohort $20.3 \%$ and $14.7 \%$, respectively [25]. The diminished intrinsic factor (IF) secretion as a result of decreased volume of IF-producing parietal cells after LSG may lead to $\mathrm{B}_{12}$ malabsorption. Severity of vitamin $\mathrm{B}_{12}$ malabsorption may be partially dependent on the size of the resected stomach. However, the volume of the specimen is not standardised. In RYGB procedure exclusion of the stomach, duodenum and proximal jejunum leads to a decreased binding with IF and a reduction of absorption surface.

Additionally, up to $35 \%$ of RYGB patients have bacterial overgrowth in the small intestine after operation that leads to $\mathrm{B}_{12}$ deficiencies as reported by Majumder et al. [26]. According to his review, the most effective route of $\mathrm{B}_{12}$ supplementation is an intramuscular injection. In our centre, supplementation was based on sublingual administration that is not dependent on IF secretion and absorption from the ileum. We also believe that it improves patients comfort and increases compliance, as it is a non-invasive, more comfortable than intramuscular method of vitamin supplementation. A recent study by Bensky et al. which compared sublingual with intramuscular method of vitamin $\mathrm{B}_{12}$ supplementation in deficient patients concluded that sublingual application is sufficient or even superior to the latter [27]. Other routes of vitamin 
Table 3 Percentage of patients with deficiencies

\begin{tabular}{|c|c|c|c|c|}
\hline Variable & Type of operation & Baseline (\%) & 1 month (\%) & 12 months (\%) \\
\hline \multirow[t]{2}{*}{ Erythrocytes $\left[10^{12} / \mathrm{L}\right]$} & RYGB & 4.3 & 4.5 & 2.1 \\
\hline & LSG & 0.0 & 2.3 & 0.0 \\
\hline \multirow[t]{2}{*}{ Haemoglobin [g/dL] } & RYGB & 21.3 & 38.6 & 27.7 \\
\hline & LSG & 15.7 & 20.9 & 15.7 \\
\hline \multirow[t]{2}{*}{ Iron $[\mu \mathrm{mol} / \mathrm{L}]$} & RYGB & 4.3 & 15.9 & 8.5 \\
\hline & LSG & 5.9 & 11.4 & 2.0 \\
\hline \multirow[t]{2}{*}{ Ferritin $[\mathrm{pg} / \mathrm{L}]$} & RYGB & 8.5 & 0.0 & $17.0^{+}$ \\
\hline & LSG & 11.8 & $0.0^{*}$ & $17.6^{+}$ \\
\hline \multirow[t]{2}{*}{ Transferrin $[\mathrm{g} / \mathrm{L}]$} & RYGB & 4.3 & $63.6^{*}$ & $8.5^{+}$ \\
\hline & LSG & 7.8 & $62.8^{*}$ & $27.5^{*^{+}}$ \\
\hline \multirow[t]{2}{*}{ Total protein $[\mathrm{g} / \mathrm{L}]$} & RYGB & 2.1 & $22.0 *$ & $4.3^{+}$ \\
\hline & LSG & 3.9 & $34.9^{*}$ & $3.9^{+}$ \\
\hline \multirow[t]{2}{*}{ Albumin $[\mathrm{g} / \mathrm{L}]$} & RYGB & 2.1 & 2.6 & 4.3 \\
\hline & LSG & 19.6 & $2.3^{*}$ & $3.9^{*}$ \\
\hline \multirow[t]{2}{*}{ Magnesium $[\mathrm{mmol} / \mathrm{L}]$} & RYGB & 4.3 & 9.1 & 4.3 \\
\hline & LSG & 7.8 & 6.8 & 2.0 \\
\hline \multirow[t]{2}{*}{ Calcium $[\mathrm{mmol} / \mathrm{L}]$} & RYGB & 6.4 & 6.8 & 4.3 \\
\hline & LSG & 7.8 & 2.3 & 3.9 \\
\hline \multirow[t]{2}{*}{ Folic acid [nmol/L] } & RYGB & 12.8 & 11.4 & 6.4 \\
\hline & LSG & 0.0 & 2.3 & $13.7^{*+}$ \\
\hline \multirow[t]{2}{*}{$\operatorname{Vitamin} \mathrm{B}_{12}[\mathrm{nmol} / \mathrm{L}]$} & RYGB & 6.4 & 2.3 & $25.5^{*^{+}}$ \\
\hline & LSG & 5.9 & 2.3 & 7.8 \\
\hline
\end{tabular}

*Statistically significant difference $(p<0.05)$ versus baseline values

${ }^{+}$Statistically significant difference $(p<0.05) 1$-month vs 12 -month values

$R Y G B$, Roux-Y gastric bypass; $L S G$, laparoscopic sleeve gastrectomy
$\mathrm{B}_{12}$ administration were also reported. Sarker et al. suggested that patients with impaired coagulation or simultaneous vitamin K deficiency should be supplemented with oral, nasal or sublingual vitamin $\mathrm{B}_{12}$ [28].

As Stein et al. reported in the review article, postsurgical vitamin $\mathrm{B}_{12}$ deficiency leading to anaemia occurs much more often in RYGB cohort than in LSG cohort. Similarly to our results, $\mathrm{B}_{12}$ deficiency was much more common pre- and postoperatively in the RYGB group. Stein et al. suggested to prescribe high dose intramuscular or subcutaneous $\mathrm{B}_{12}$ supplementation in the malabsorptive cohort, but not after restrictive procedures [29].

Iron deficiency may be present in obese patients prior to operation due to constant inflammatory state. One of the underlying mechanisms may be the production of hepcidin which decreases serum iron level. Bariatric operations may exacerbate it [30]. In Peterson et al. [8] study, frequency of iron deficiency in the group of RYGB candidates reached $36.2 \%$ which is lower than observed in our cohort.

Prevalence of albumin deficiency in the RYGB group was low preoperatively, similarly to results reported by Gobato et al. [31]. However, albumin levels remained unchanged during 12 months after surgery contrary to some other reports.
Nicoletti et al. showed a significant increase in albumin concentrations in patients after RYGB [32].

Due to possible nutritional complications resulting from bariatric surgery, it is important to provide complex screening and prevention of nutritional deficiencies [33]. In a clinical report on nutritional and pharmacologic challenges after bariatric surgeries, Lizer et al. recommended that all these patients should receive lifelong vitamin and mineral supplementation postoperatively. However, there are also opinions that only RYGB cohort should receive this form of prevention [34]. There are multiple strategies regarding type and complexity of postoperative supplementation. Via et al. suggests that lifelong screening and multivitamin, calcium and vitamin $\mathrm{D}$ supplementation is a general recommendation for all patients after bariatric operations [35]. Kwon et al. claims that supplementation of only selected micronutrients (vitamin $\mathrm{B}_{12}$ and iron) may not always be sufficient in deficient patients [36]. Customised supplementation formula compared to a standard multivitamin product in RYGB cohort may lead to improved reduction of nutritional deficiencies [37]. From our perspective, it may be difficult to accurately assess the efficiency of supplementation regimen due to unknown patients' compliance; therefore, we suggest both general and specific 
micronutrient supplementation, especially in the early postoperative phase. Long-term follow-up should also include systematic periodical assessment of laboratory findings and supplementation adjusted to individual patients' needs.

\section{Limitations}

The limitations of our study included its retrospective character and incomplete information regarding compliance with supplementation regimen. The early follow-up assessment was not completed by all the participants and postoperative attrition might have influenced reported outcomes. The study aim was designed to analyse nutritional deficiencies during 12-month follow-up which is commonly considered an early period after bariatric surgery and does not allow to draw definite conclusions on the risk of deficiencies in a long term.

\section{Conclusions}

Nutritional deficiencies may occur regardless of the type of bariatric operation; therefore, it is crucial to regularly control the patients' nutritional parameters during the follow-up. Bariatric surgery may exacerbate malnutrition; therefore, we recommend routine preoperative and lifelong postoperative laboratory testing for deficiencies in RYGB and LSG cohort and an appropriate individualised oral supplementation.

Both bariatric procedures may lead to malnutrition regardless of malabsorptive or restrictive properties. Possible adjustments in postoperative supplementation regimen based on our results include an increase in doses of vitamin $B_{12}$ in RYGB patients and folic acid supplementation in LSG patients. However, nutritional requirements change during different periods after surgery and in order to create recommendations better reflecting patients' needs, long-term follow-up results should be analysed.

\section{Compliance with Ethical Standards}

Conflict of Interest The authors declare that they have no conflict of interests.

Statement of Informed Consent Due to the retrospective design of this study, formal consent was not required; however, preoperatively, all patients provided written informed consent for their operation.

Statement of Human and Animal Rights All procedures performed in studies involving human participants were in accordance with the standards of the institution and/or national research committee and with the 1964 Helsinki declaration and its later amendments or comparable ethical standards.
Open Access This article is distributed under the terms of the Creative Commons Attribution 4.0 International License (http:// creativecommons.org/licenses/by/4.0/), which permits unrestricted use, distribution, and reproduction in any medium, provided you give appropriate credit to the original author(s) and the source, provide a link to the Creative Commons license, and indicate if changes were made.

\section{References}

1. Mingrone G, Panunzi S, De Gaetano A, et al. Bariatric surgery versus conventional medical therapy for type 2 diabetes. N Engl J Med. 2012;366(17):1577-85.

2. Ponce J, DeMaria EJ, Nguyen NT, et al. American Society for Metabolic and Bariatric Surgery estimation of bariatric surgery procedures in 2015 and surgeon workforce in the United States. Surg Obes Relat Dis. 2016;12(9):1637-9.

3. Buchwald H, Williams SE. Bariatric surgery worldwide 2003. Obes Surg. 2004;14(9):1157-64.

4. Gagner M, Rogula T. Laparoscopic reoperative sleeve gastrectomy for poor weight loss after biliopancreatic diversion with duodenal switch. Obes Surg. 2003;13(4):649-54.

5. Fried M, Hainer V, Basdevant $\mathrm{A}$, et al. Inter-disciplinary European guidelines on surgery of severe obesity. Int J Obes. 2007;31:56977.

6. Allied Health Sciences Section Ad Hoc Nutrition Committee, Aills L, Blankenship J, et al. ASMBS allied health nutritional guidelines for the surgical weight loss patient. Surg Obes Relat Dis. 2008;4(5 Suppl):S73-108.

7. Bailly L, Schiavo L, Sebastianelli L, et al. Anemia and bariatric surgery: results of a National French Survey on administrative data of 306,298 consecutive patients between 2008 and 2016. Obes Surg. 2018;28(8):2313-232.

8. Gehrer S, Kern B, Peters T, et al. Fewer nutrient deficiencies after laparoscopic sleeve gastrectomy (LSG) than after laparoscopic Roux-Y-gastric bypass (LRYGB)-a prospective study. Obes Surg. 2010;20(4):447-53.

9. Alexandrou A, Armeni E, Kouskouni E, et al. Cross-sectional longterm micronutrient deficiencies after sleeve gastrectomy versus Roux-en-Y gastric bypass: a pilot study. Surg Obes Relat Dis. 2014;10(2):262-8.

10. Coupaye $\mathrm{M}$, Rivière $\mathrm{P}, \mathrm{Breuil} \mathrm{MC}$, et al. Comparison of nutritional status during the first year after sleeve gastrectomy and Roux-en-Y gastric bypass. Obes Surg. 2014;24(2):276-83.

11. Leyba JL, Llopis SN, Aulestia SN. Laparoscopic Roux-en-Y gastric bypass versus laparoscopic sleeve gastrectomy for the treatment of morbid obesity. A prospective study with 5 years of follow-up. Obes Surg. 2014;24(12):2094-8.

12. Peterson LA, Cheskin LJ, Furtado M, et al. Malnutrition in bariatric surgery candidates: multiple micronutrient deficiencies prior to surgery. Obes Surg. 2016;26(4):833-8.

13. Muñoz M, Botella-Romero F, Gómez-Ramírez S, et al. Iron deficiency and anaemia in bariatric surgical patients: causes, diagnosis and proper management. Nutr Hosp. 2009;24(6):640-54.

14. Jáuregui-Lobera I. Iron deficiency and bariatric surgery. Nutrients. 2013;5(5):1595-608

15. Gerber P, Anderin C, Thorell A. Weight loss prior to bariatric surgery: an updated review of the literature. Scand J Surg. 2015;104(1):33-9.

16. Van der Beek ES, Monpellier VM, Eland I, et al. Nutritional deficiencies in gastric bypass patients; incidence, time of occurrence and implications for post-operative surveillance. Obes Surg. 2015;25(5):818-23. 
17. Manning S, Carter NC, Pucci A, et al. Age- and sex-specific effects on weight loss outcomes in a comparison of sleeve gastrectomy and Roux-en-Y gastric bypass: a retrospective cohort study. BMC Obes. 2014;1:12.

18. Kang JH, Le QA. Effectiveness of bariatric surgical procedures: a systematic review and network meta-analysis of randomized controlled trials, Hsu. C-S, ed. Medicine. 2017;96(46):e8632.

19. Liu C, Wu D, Zhang JF, et al. Changes in bone metabolism in morbidly obese patients after bariatric surgery: a meta-analysis. Obes Surg. 2016;26(1):91-7.

20. Hage MP, El-Hajj Fuleihan G. Bone and mineral metabolism in patients undergoing Roux-en-Y gastric bypass. Osteoporos Int. 2014;25(2):423-39.

21. Brolin RE, Gorman JH, Gorman RC, et al. Prophylactic Iron supplementation after Roux-en-Y gastric bypass: a prospective, double-blind, randomized study. Arch Surg. 1998;133(7):740-4.

22. Kotkiewicz A, Donaldson K, Dye C, et al. Anemia and the need for intravenous iron infusion after Roux-en-Y gastric bypass. Clin Med Insights Blood Disord. 2015;8:9-17.

23. Varma S, Baz W, Badine E, et al. Need for parenteral iron therapy after bariatric surgery. Surg Obes Relat Dis. 2008;4(6):715-9.

24. von Drygalski A, Andris DA. Anemia after bariatric surgery: more than just Iron deficiency. Nutr Clin Pract. 2009;24(2):217-26.

25. Lefebvre $P$, Letois $F$, Sultan A, et al. Nutrient deficiencies in patients with obesity considering bariatric surgery: a cross-sectional study. Surg Obes Relat Dis. 2014;10(3):540-6.

26. Majumder S, Soriano J, Louie Cruz A, et al. Vitamin $B_{12}$ deficiency in patients undergoing bariatric surgery: preventive strategies and key recommendations. Surg Obes Relat Dis. 2013;9(6):1013-9.

27. Bensky MJ, Ayalon-Dangur I, Ayalon-Dangur R, et al. Comparison of sublingual vs. intramuscular administration of vitamin B12 for the treatment of patients with vitamin $\mathrm{B}_{12}$ deficiency. Drug Deliv Transl Res. 2019;9:625-30.

28. Sarker A, Meek CL, Park A. Biochemical consequences of bariatric surgery for extreme clinical obesity. Ann Clin Biochem. 2016;53(Pt $1: 21-31$.
29. Stein J, Stier C, Raab H, et al. Review article: the nutritional and pharmacological consequences of obesity surgery. Aliment Pharmacol Ther. 2014;40(6):582-609.

30. Gletsu-Miller N, Wright BN. Mineral malnutrition following bariatric surgery. Adv Nutr. 2013;4(5):506-17.

31. Gobato RC, Seixas Chaves DF, Chaim EA. Micronutritient and physiologic parameters before and 6 months after RYGB. Surg Obes Relat Dis. 2014;10(5):944-51.

32. Nicoletti CF, Morandi Junqueira-Franco MV, dos Santos JE, et al. Protein and amino acid status before and after bariatric surgery: a 12-month follow-up study. Surg Obes Relat Dis. 2013;9(6):100812.

33. Stroh C, Benedix F, Meyer F, et al. Nutrient deficiencies after bariatric surgery - systematic literature review and suggestions for diagnostics and treatment. Zentralbl Chir. 2015;140(4):407-16.

34. Lizer MH, Papageorgeon H, Glembot TM. Nutritional and pharmacologic challenges in the bariatric surgery patient. Obes Surg. 2010;20(12):1654-9.

35. Via MA, Mechanick JI. Nutritional and micronutrient care of bariatric surgery patients: current evidence update. Curr Obes Rep. 2017;6(3):286-96.

36. Kwon Y, Kim HJ, Lo Menzo E, et al. Anemia, iron and vitamin B12 deficiencies after sleeve gastrectomy compared to Roux-en-Y gastric bypass: a meta-analysis. Surg Obes Relat Dis. 2014;10(4):58997.

37. Dogan K, Aarts EO, Koehestanie P, et al. Optimization of vitamin supplementation after Roux-en-Y gastric bypass surgery can lower postoperative deficiencies: a randomized controlled trial. Medicine (Baltimore). 2014;93(25):e169.

Publisher's Note Springer Nature remains neutral with regard to jurisdictional claims in published maps and institutional affiliations. 